\title{
Outbreak of KPC-2-producing Klebsiella pneumoniae endowed with ceftazidime-avibactam resistance mediated through a VEB-1-mutant (VEB-25), Greece, September to October 2019
}

Irene Galani ${ }^{1}$, Ilias Karaiskos ${ }^{2}$, Maria Souli ${ }^{1}$, Vassiliki Papoutsaki ${ }^{3}$, Lamprini Galani², Aikaterini Gkoufa², Anastasia Antoniadou ${ }^{1}$, Helen Giamarellou ${ }^{2}$

1. Infectious Diseases Laboratory, 4th Department of Internal Medicine, National and Kapodistrian University of Athens, Athens, Greece

2. $1^{\text {st }}$ Internal Medicine $\&$ Infectious Diseases Department, Hygeia General Hospital, Athens, Greece

3. Infectious Diseases Laboratory, Hygeia General Hospital, Athens, Greece

Correspondence: Irene Galani (egalani@med.uoa.gr)

Citation style for this article:

Galani Irene, Karaiskos Ilias, Souli Maria, Papoutsaki Vassiliki, Galani Lamprini, Gkoufa Aikaterini, Antoniadou Anastasia, Giamarellou Helen. Outbreak of KPC-2producing Klebsiella pneumoniae endowed with ceftazidime-avibactam resistance mediated through a VEB-1-mutant (VEB-25), Greece, September to October 2019. Euro Surveill. 2020;25(3):pii=2000028. https://doi.org/10.2807/1560-7917.ES.2020.25.3.2000028

From September to October 2019, seven patients colonised or infected with a ceftazidime-avibactam (CZA)-resistant Klebsiella pneumoniae carbapenemase (KPC)-2-producing $K$. pneumoniae were detected in two intensive care units of a Greek general hospital. The outbreak strain was sequence type (ST)147 and co-produced KPC-2 and the novel plasmid-borne Vietnamese extended-spectrum $\beta$-lactamase (VEB)-25 harbouring a K234R substitution associated with CZA resistance. Epidemiological investigations revealed that the resistance was probably acquired by horizontal transmission independently from previous CZA exposure.

The spread of carbapenemase-producing Klebsiella pneumoniae (CPKP) has become a significant problem worldwide. Ceftazidime-avibactam (CZA) is a novel $\beta$-lactam $/ \beta$-lactamase inhibitor combination effective against strains producing serine carbapenemases, including $K$. pneumoniae carbapenemase (KPC)- and oxacillinase (OXA)-48-type enzymes [1]. Resistance to CZA has already been described and was mainly linked to specific mutations in bla ${ }_{\mathrm{KPC}}$ [2], alterations in Ompk35 and Ompk36 porins and/or increased expression of bla ${ }_{\text {KPC }}$ [3], as well as amino acid substitutions in cefotaxime-M $\beta$-lactamase (CTX-M)-14 [4]. Moreover during editing of this report, two $K$. pneumoniae isolates resistant to CZA were reported from Greece due to Vietnamese extended-spectrum $\beta$-lactamase (VEB)-25 [5]. The European Centre for Disease Prevention and Control (ECDC) has identified CZA resistance as an important cross-border threat that merits careful monitoring [6]. In this article, we report an outbreak caused by a CZA-resistant $K$. pneumoniae strain in a hospital in Athens, Greece in 2019. The outbreak molecular investigation revealed that resistance was due to plasmid-borne VEB-25, which differs from VEB-1 by one mutation. The affected patients had not previously been treated with CZA. Another isolate detected in the same hospital in 2018 had a different bla ${ }_{\text {VEB-1 }}$ variant leading to CZA-resistance but this variant did not belong to the outbreak clone.

\section{Outbreak detection and investigation}

The index hospital comprises two mixed intensive care units (ICU) and one high dependency unit (HDU) that are located in different areas of the hospital. ICU-1 includes a 14 -bed open space unit and four singlepatient rooms. ICU-2 consists of seven single-patient rooms, whereas HDU has 12 beds, and one isolation room. Rectum surveillance cultures are performed routinely twice weekly in all ICU patients and screened for carbapenemase producers. In November 2018, 11 months after the introduction of CZA in clinical practice (December 2017), one patient hospitalised in the HDU was found to be colonised by a CZA-resistant KPC-producing $K$. pneumoniae strain (KP121) after CZA treatment (Table 1 and Table 2). The patient was isolated in a single room under strict contact precautions until discharge and no more cases were detected. Since then, all surveillance strains that were KPC-producers have been routinely evaluated for CZA resistance. This practice enabled the early detection of the outbreak. From September 2019 to October 2019, four patients in ICU-1 and three patients in ICU-2 were found to be colonised by a CZA-resistant KPC-producing K. pneumoniae strain and three of them developed an infection (Table 2). The outbreak prompted an epidemiological and molecular investigation. Prevention efforts including intensification of contact precautions (provision 
TABLE 1

Characteristics of the ceftazidime-avibactam-resistant Klebsiella pneumoniae strains detected in a general hospital as well as their transconjugants and one previously characterised transconjugant producing VEB-1, Greece, 2018 and 2019 ( $\mathrm{n}=6$ strains)

\begin{tabular}{|c|c|c|c|c|c|c|}
\hline Isolate & $\begin{array}{c}\text { K. pneumoniae } \\
\text { ST39 strain } \\
\left(\mathrm{KP}_{121}\right)\end{array}$ & $\begin{array}{c}\text { E. coli }{ }^{\mathrm{b}} \\
\text { RC85-pl121 }\end{array}$ & $\begin{array}{c}\text { K. pneumoniae } \\
\text { ST147 outbreak strain } \\
\text { (KP67585) }\end{array}$ & $\begin{array}{c}\text { E. colic } \\
\text { RC85-pl67585 }\end{array}$ & $\begin{array}{c}\text { E. colid } \\
\text { RC85-pl52 }\end{array}$ & $\begin{array}{l}\text { E. coli } \\
\text { RC85 }\end{array}$ \\
\hline \multicolumn{7}{|l|}{ Type } \\
\hline KPC-type & KPC-2 & None & $\mathrm{KPC}-2$ & None & None & None \\
\hline VEB-type & VEB-14 & VEB-14 & VEB-25 & VEB-25 & VEB-1 & None \\
\hline Antibiotics tested & \multicolumn{6}{|c|}{ Minimum inhibitory concentration in $\mathrm{mg} / \mathrm{L}$} \\
\hline Ampicillin-sulbactam & $>16$ & $>16$ & \begin{tabular}{|c|}
$>16$ \\
\end{tabular} & $>16$ & $>16$ & $\leq 2$ \\
\hline Piperacillin-tazobactam & $>64$ & 32 & $>64$ & $>64$ & $>64$ & $\leq 4$ \\
\hline Cefoxitin & $>32$ & $\leq 4$ & $>32$ & $\leq 4$ & $\leq 4$ & $\leq 4$ \\
\hline Ceftazidime & 2,048 & 4,096 & 1,024 & 512 & 512 & 0.25 \\
\hline Ceftazidime-avibactam & 64 & 256 & 64 & 16 & 0.25 & 0.25 \\
\hline Ceftriaxone & 132 & 32 & 132 & 8 & 32 & $\leq 1$ \\
\hline Cefepime & 132 & 132 & 132 & 2 & 2 & $\leq 1$ \\
\hline Aztreonam & 132 & 132 & 132 & 132 & $>32$ & $\leq 1$ \\
\hline Imipenem & 64 & 0.12 & 32 & 0.12 & 0.12 & 0.12 \\
\hline Imipenem-relebactam & 0.5 & 0.12 & 0.5 & 0.12 & 0.12 & 0.12 \\
\hline Meropenem & $>64$ & 0.06 & 64 & 0.06 & 0.06 & 0.06 \\
\hline Meropenem-vaborbactam & 0.5 & 0.06 & 0.25 & 0.06 & 0.06 & 0.06 \\
\hline Amikacin & 132 & 132 & 132 & 132 & 132 & $\leq 2$ \\
\hline Gentamicin & $>8$ & $>8$ & $>8$ & $>8$ & $>8$ & $\leq 1$ \\
\hline Ciprofloxacin & $>2$ & $\leq 0.25$ & 12 & $\leq 0.25$ & $\leq 0.25$ & $\leq 0.25$ \\
\hline Levofloxacin & $>4$ & $\leq 0.12$ & $>4$ & $\leq 0.12$ & $\leq 0.12$ & $\leq 0.12$ \\
\hline Tigecycline & 24 & 1 & $2->4$ & 2 & $\leq 0.5$ & $\leq 0.5$ \\
\hline Fosfomycin & 64 & $\leq 16$ & 128 & $\leq 16$ & $\leq 16$ & $\leq 16$ \\
\hline Colistin & 2 & 0.5 & 64 & 0.5 & 0.5 & 0.5 \\
\hline Trimethoprim-sulfamethoxazole ${ }^{\mathrm{e}}$ & $>8$ & $>8$ & $>8$ & $>8$ & $>8$ & $\leq 1$ \\
\hline Chloramphenicol & $>128$ & 128 & $>128$ & 32 & 32 & 8 \\
\hline \multicolumn{7}{|l|}{ Other $\beta$-lactamase genes } \\
\hline$\beta$-lactamase genes & $\begin{array}{l}b_{\mathrm{SHV}-11}, \\
\text { bla }_{\mathrm{OXA}-10} \\
\text { bla }_{\mathrm{TEM}-1 \mathrm{~B}}\end{array}$ & $\begin{array}{l}b a_{\mathrm{OXA}-10} \\
\text { bla }_{\mathrm{TEM}-1 \mathrm{~B}}\end{array}$ & $\begin{array}{l}b_{\mathrm{SHV}-11}, \\
\text { bla }_{\mathrm{OXA}-10}, \\
\text { bla }_{\mathrm{TEM-1 \textrm {B }}}\end{array}$ & $\begin{array}{l}b a_{\mathrm{OXA}-10} \\
b a_{\mathrm{TEM}-1 \mathrm{~B}}\end{array}$ & $\begin{array}{l}b a_{\mathrm{OXA}-10} \\
\text { bla }_{\mathrm{TEM}-1 \mathrm{~B}}\end{array}$ & None \\
\hline \multicolumn{7}{|l|}{ Major porin mutation } \\
\hline OmpK35 & WT & ND & PSC_aa173 & ND & ND & ND \\
\hline $\mathrm{OmpK}_{3} 6$ & v3 variant & ND & v3 variant & ND & ND & ND \\
\hline $\mathrm{OmpK}_{37}$ & PSC_aa251 & ND & WT & ND & ND & ND \\
\hline
\end{tabular}

E. coli: Escherichia coli; K. pneumoniae: Klebsiella pneumoniae; KPC: K. pneumoniae carbapenemase; MIC: minimum inhibitory concentration; ND: not determined; OmpK35: OmpK35-WT (GenBank accession number: GU460162); OmpK36: OmpK36_v3 (GenBank accession number: JQ781655); OmpK37: OmpK37-WT (GenBank accession number: WP 002902433); PSC: premature stop codon; PSC aa173: premature stop codon at amino acid 173; PSC_aa251: premature stop codon at amino acid 251; ST: sequence type; VEB: Vietnamese extended-spectrum $\beta$-lactamase; WT: wild type.

a The isolates detected in the general hospital, which are presented in the table, include one isolate (KP121) producing VEB-14 (ST39) that was discovered in 2018, as well as one isolate (KP67585) producing VEB-25 (ST147) that represents a strain responsible for the 2019 hospital outbreak, which is described in this report.

b Transconjugant $E$. coli RC85 isolate harbouring bla ${ }_{\mathrm{VEB}-14}$ carrying plasmid.

'Transconjugant $E$. coli RC85 isolate harbouring bla ${ }_{\mathrm{VEB}-25}$ carrying plasmid.

${ }^{\mathrm{d}}$ Transconjugant $E$. coli RC85 isolate harbouring bla ${ }_{\mathrm{VEB}-1}$ carrying plasmid. The donor isolate was an OXA-48-producing K.pneumoniae isolate carrying an IncA/C2 plasmid.

e Trimethoprim-sulfamethoxazole in the ratio 1:19. MICs are expressed as the trimethoprim concentration. 
of personal protective equipment outside the patient room and use of gloves and gown upon entering the room, minimising risk of environmental contamination by dressing patients in a gown during transport and applying all standard precautions at the receiving unit, dedicating noncritical items for single patient use and having dedicated infection control nurses overseeing strict implementation of measures), isolation of colonised patients in single rooms and strict hand hygiene practices successfully contained the outbreak as no new case was identified after October 2019.

\section{Microbiological and molecular analyses}

Susceptibility testing performed by VITEK 2, broth microdilution and minimum inhibitory concentration (MIC) test strips revealed resistance, according to the European Committee on Antimicrobial Susceptibility Testing (EUCAST) clinical breakpoints (2019, v 9.0) [7], to all antimicrobial agents tested except imipenem-relebactam, meropenem-vaborbactam, and colistin. CZA MICs (tested with a fixed avibactam concentration of $4 \mathrm{mg} / \mathrm{L}$ ) ranged from 32 to $64 \mathrm{mg} / \mathrm{L}$ (Supplementary Table 1). PCR and sequencing analysis [8], showed that all isolates harboured bla ${ }_{\mathrm{KPC}-2}$ as the sole carbapenemase gene. In addition to KPC-2, all eight isolates produced sulfhydryl reagent variable $\beta$-lactamase (SHV)-11, OXA10, Temoniera $\beta$-lactamase (TEM)-1 and an extended spectrum $\beta$-lactamase (ESBL) of VEB-type. Isolate KP121 produced VEB-14 (T216del, per Ambler numbering scheme) [9]. All other isolates produced a novel VEB-1 variant carrying a substitution of lysine by an arginine at position 237 (K234R, per Ambler numbering scheme), due to nt A710G substitution (Supplementary Table 1). The mutant bla ${ }_{\text {VEB-K } 237 R}$ sequence was assigned the novel allele VEB-25 (GenBank accession number: MN853159). Further acquired resistance genes justifying the resistance phenotype of studied strains are presented in Supplementary Table 1.

Whole genome sequencing (WGS) analysis, performed as described previously [10], revealed that both bla ${ }_{4}$ and bla ${ }_{\text {VEB-25 }}$ were carried on conjugative plasmids of InCA/C2 incompatibility group. bla ${ }_{V E B 14}$ and bla were present as the first gene cassette of an integron that also included an array of aadB, arr2, $\mathrm{cm} l \mathrm{~A}_{1}$, bla OXA. $_{\text {. }}$ ${ }_{10}$, and $\operatorname{aad} A_{1}$ cassettes. An insertion sequence (IS) 1999 was located upstream of both bla ${ }_{V E B}$-variants providing a strong promoter for bla ${ }_{\text {VEB }}$ expression. The two whole genome sequences were deposited in the Sequence Read Archive (SRA) under the following accession numbers: PRJNA602658 (for the bla VEB. ${ }_{14}$ harbouring strain) and PRJNA602657 (for the strain with bla VEB-25 $_{2}$.

PFGE analysis classified the studied isolates in two pulsotypes [8], with $<80 \%$ Dice similarity index. The first pulsotype included only the $\mathrm{KP}_{121}$, isolated in November 2018, while all the strains isolated in the period September-October 2019, belonged to one clone (second pulsotype). Based on WGS [10], KP121 belonged to sequence type (ST)39 and capsular type
KL23 (wzc: 24; wzi: 83), while the outbreak strain belonged to clonal lineage ST147 and capsular type KL64 (wzC: 64; wzi: 64).

The ST147 outbreak strain, which was similar to one of the strains reported recently from another hospital in Athens [5], harboured a non-functional porin $\mathrm{OmpK}_{35}$ due to a premature stop codon at position 173, while ST39 isolate (KP121) had an intact OmpK35. OmpK36 v3 variant, previously associated with ST147 K. pneumoniae isolates from Greece [11], was present in both ST147 and ST39 isolates. OmpK36_v3 harbours a duplication of two amino acids, Gly134-Asp135, located at the conserved loop $\mathrm{L}_{3}$, which contributes to high-level resistance to carbapenems [11]. However, OmpK35 and $\mathrm{OmpK}_{3} 6$ are not the primary pathways for avibactam into the cell of $K$. pneumoniae [12].

bla $_{\text {VEB }}$-harbouring plasmids from the ST39 (KP121), and the ST147 outbreak strain (KP67585) were transferred by conjugation to rifampicin-resistant Escherichia coli RC85 R K12. The susceptibility profile of the transconjugants (RC85/pli21 and RC85/pl67585) is shown in Table 1. The susceptibility profile of a previously studied transconjugant, RC $85 / \mathrm{pl}_{52}$, harbouring bla VEB-1 $_{1}$ is included in Table 1 for comparison. Transconjugants were resistant to $\beta$-lactams, aminoglycosides, trimethoprim/sulfamethoxazole and chloramphenicol (Table 1). PCR and sequencing confirmed the presence of bla ${ }_{\text {VEE }}$, bla OXA-10, bla ${ }_{\text {TEM-1B }}$ and $r m t B 1$ and the absence of bla ${ }_{\mathrm{KPC}-2}$ in all transconjugants. CZA MICs determined by Liofilchem MIC Test Strips, differed between the transconjugants depending on the VEB-variant they produced. RC $85 / \mathrm{pl} 52$ harbouring bla ${ }_{\text {VEB-1 }}$ exhibited ceftazidime MIC of $512 \mathrm{mg} / \mathrm{L}$, which was reduced to $0.25 \mathrm{mg} / \mathrm{L}$ (2,048-fold reduction) in the presence of avibactam. Transconjugant RC85/ pl67585 carrying bla ${ }_{\text {VEB-2. }}$, exhibited similar MIC to ceftazidime $(512 \mathrm{mg} / \mathrm{L})$, which was reduced to $16 \mathrm{mg} / \mathrm{L}$ by avibactam (32-fold reduction). Transconjugant RC85/ pl121 carrying bla ${ }_{\text {VEB-14 }}$, exhibited higher MIC to ceftazidime $(4,096 \mathrm{mg} / \mathrm{L})$ and to CZA $(256 \mathrm{mg} / \mathrm{L})(16$-fold reduction by avibactam).

\section{Epidemiological investigation}

The eight patients found with a CZA-resistant KPC producing $K$. pneumoniae comprised five men and three women. Possible chains of transmission were investigated but no common source was identified between patients in ICU-1 and ICU-2. All colonised or infected patients had a long period of ICU stay (median: 48 days; range: $11-56$ days) before colonisation (Figure). All patients were originally hospitalised in the index hospital with the exception of one patient (KP368), colonised with the outbreak strain, who had been hospitalised in another hospital for 24 hours. None had been previously received CZA, however all patients had been pre-treated or were on $\beta$-lactam therapy during hospitalisation in the ICU (Table 2). Only the patient with $\mathrm{KP}_{121}$ had received CZA before colonisation but he represented an independent case not 


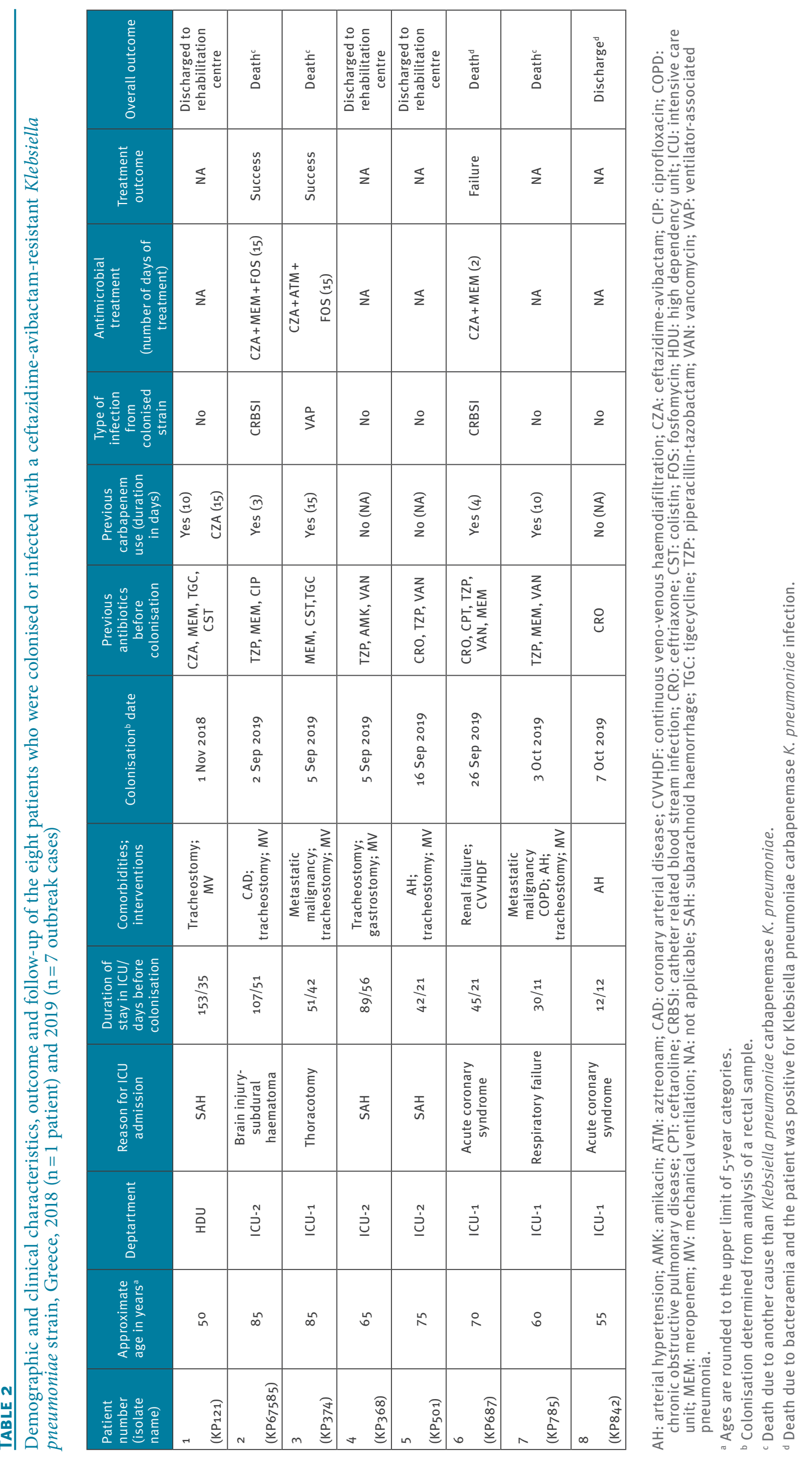


Timeline of the hospital outbreak of sequence type (ST)147 Klebsiella pneumoniae harbouring a Vietnamese extendedspectrum $\beta$-lactamase-25, Greece, 2019 ( $n=7$ outbreak cases)

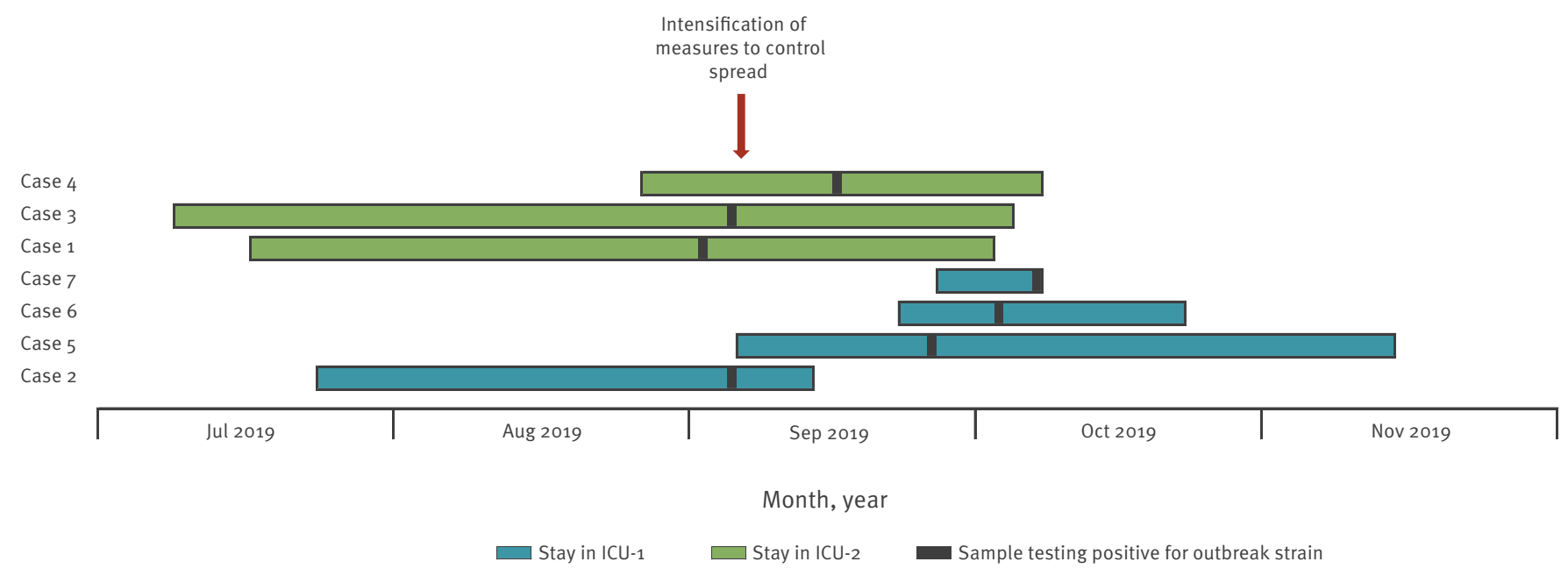

Cases 1, 2, 3, 4, 5, 6 and 7 correspond to patients 2, 3, 4, 5, 6, 7 and 8 respectively in Table 2.

Intensified measures included the requirement of wearing gloves and a gown upon entering the case's room, minimal transport of this patient and, if so, prior advising the receiving unit so that necessary precautions could be taken in advance, dedicated use of noncritical items and having dedicated infection control nurses inspecting the implementation of infection control measures, isolation of colonised patients in single rooms and strict hand hygiene practices.

related to the outbreak. Three patients developed an infection: two catheter-related bloodstream infections (catheter culture revealed the same pathogen in both cases) and one ventilator-associated pneumonia. The salvage therapeutic regimens were chosen based on in vitro data showing synergy of the combinations used, all including CZA, the rationale being that avibactam inactivates the class $A, C$, or D $\beta$-lactamases and restores susceptibility to aztreonam or meropenem [13-15]. The triple combination was successful in two of the cases at Day 14, while the combination of CZA and meropenem was reported as failure in the remaining case (Table 2); in terms of all-cause mortality, by Day 28 all infected patients had died.

\section{Discussion}

CZA demonstrates high in vitro activity against nonmetallo- $\beta$-lactamase-producing $K$. pneumoniae strains in Greece [8]. To date, there are two reports on sporadic cases of CZA resistant $K$. pneumoniae from Greece, due to the production of KPC-23 [10], or VEB-25 [5]. In the present study, we provide further evidence that CZA resistance may emerge through evolution of bla ${ }_{\mathrm{VEB}-1}$ with the detection of a bla ${ }_{\mathrm{VEB}-25}$-harbouring strain causing a hospital outbreak and another strain endowed with bla $a_{\text {VEB-14 }}$ in a single hospitalised patient. Both VEB-14 and VEB-25 variants exhibit decreased inactivation by avibactam.

The $K$. pneumoniae isolates presented in this study produced KPC-2, VEB-14 or VEB-25, OXA-10, TEM-1B and the chromosomally encoded SHV-11 $\beta$-lactamases. The transconjugants carried bla ${ }_{\text {OXA-10 }}$, bla ${ }_{\text {TEM-1B }}$, and bla ${ }_{1}$, or bla $a_{\mathrm{VEB}-14}$ or bla ${ }_{\mathrm{VEB}-25}$. Avibactam appeared to reduce ceftazidime MICs to a lesser extent in the presence of VEB-14 (16-fold reduction) or VEB-25 (32-fold reduction) than in the presence of VEB-1 (2,048-fold reduction).

VEB-25, which was recently described [5], harbours a substitution of lysine by an arginine at position 237 (K234R, per Ambler numbering scheme), due to $\mathrm{nt}$ A710G substitution, while VEB-14 [9], has a threonine deletion at position 217 (T216del, per Ambler numbering scheme).

K234 residue is highly conserved among class $A$ $\beta$-lactamases (Supplementary Figure 1), forming strong hydrogen bonds with the sulphate group of avibactam [9]. We hypothesise that K234R substitution resulted in a structural change that attenuated avibactam's inhibitory effect by disrupting its ability to bind at the active site, thereby causing resistance. According to PappWallace et al., residue $\mathrm{K} 234$ contributes notably to the inactivation of KPC-2 by avibactam [16], while substitution K234R in SHV enzymes has been reported to lead to resistance to inhibitors [17-20].

T216 residue, although in close proximity, has no direct interaction with avibactam [9]. In an E. coli DH5a strain carrying isogenically expressed VEB-14, avibactam reduced ceftazidime MIC from 64 to $1 \mathrm{mg} / \mathrm{L}$, and VEB-14 was inhibited by avibactam in a concentration-dependent manner [9]. We hypothesise that in KP121, CZA 
resistance was due to increased expression of VEB-14 due to an IS1999 located upstream of the gene.

Previous epidemiological analyses (data not shown) indicated that a conjugative plasmid co-harbouring bla ${ }_{\mathrm{VEB}-1}$, bla ${ }_{\mathrm{OXA}-10}$, bla ${ }_{\mathrm{TEM}-\mathrm{B}}$ and $r m t B$ is present in $8 \%$ of all bla -positive isolates in Greece [21]. This already established vehicle can potentially enhance dissemination of VEB-mediated CZA resistance in Greek hospitals. The emergence of CZA resistance dramatically limits treatment options against carbapenemase-producing Enterobacterales. In this report, infected patients received salvage combination treatment, which was successful in two of the three cases. Limitations of this study include the lack of environmental sampling during the outbreak investigation, which could have revealed potential environmental sources, and the lack of biochemical studies of VEB-14 and VEB-25 enzymes, which could have further elucidated the basis of the resistance phenotype.

In conclusion, we have shown that alterations in the ESBL VEB-1 enzyme can significantly reduce CZA susceptibility in $K$. pneumoniae co-producing KPC-2 and emergence of this resistance mechanism was independent from previous CZA exposure. Further biochemical studies are needed to reveal the basis of the resistance phenotype, conferred by the two VEBvariants. The rigorous implementation of hospital infection control precautions resulted in successful containment of the outbreak, highlighting the importance of early awareness in the fight against antimicrobial resistance.

\section{Acknowledgements}

The Authors would like to acknowledge Irene Karantani from Infectious Diseases Laboratory of Hygeia Hospital, Panagiota Adamou and Anastasia Molla from the Infectious Diseases Laboratory of the $4^{\text {th }}$ Department of Internal Medicine, for outstanding technical work and Konstantina Nafplioti for providing strain RC85/pl52.

\section{Conflict of interest}

None declared.

\section{Authors' contributions}

IG: whole genome sequencing and data analysis, manuscript writing. IK: outbreak investigation, manuscript writing. MS: manuscript writing. VP: detection and susceptibility testing of bacterial isolates. LG, AG and HG: outbreak investigation team. AA: manuscript correction. All authors contributed to the revision of the draft manuscript and approved the final version.

\section{References}

1. Karaiskos I, Galani I, Souli M, Giamarellou H. Novel $\beta$-lactam$\beta$-lactamase inhibitor combinations: expectations for the treatment of carbapenem-resistant Gram-negative pathogens. Expert Opin Drug Metab Toxicol. 2019;15(2):133-49. https:// doi.org/10.1080/17425255.2019.1563071 PMID: 30626244
2. Shields RK, Chen L, Cheng S, Chavda KD, Press EG, Snyder A, et al. Emergence of Ceftazidime-Avibactam Resistance Due to Plasmid-Borne blaKPC-3 Mutations during Treatment of Carbapenem-Resistant Klebsiella pneumoniae Infections. Antimicrob Agents Chemother. 2017;61(3):e02097-16. https:// doi.org/10.1128/AAC.02097-16 PMID: 28031201

3. Giddins MJ, Macesic N, Annavajhala MK, Stump S, Khan S, McConville TH, et al. Successive emergence of ceftazidimeavibactam resistance through distinct genomic adaptations in blaKPC-2-harboring ceftazidime-avibactam resistance through distinct genomic adaptations in blaKPC-2-harboring Klebsiella pneumoniae sequence type 307 isolates. Antimicrob Agents Chemother. 2018;62(3):e02101-17. PMID: 29263067

4. Both A, Büttner H, Huang J, Perbandt M, Belmar Campos C, Christner M, et al. Emergence of ceftazidime/avibactam non-susceptibility in an MDR Klebsiella pneumoniae isolate. J Antimicrob Chemother. 2017;72(9):2483-8. https://doi. org/10.1093/jac/dkx179 PMID: 28637339

5. Voulgari E, Kotsakis SD, Giannopoulou P, Perivolioti E, Tzouvelekis LS, Miriagou V. Detection in two hospitals of transferable ceftazidime-avibactam resistance in Klebsiella pneumoniae due to a novel VEB $\beta$-lactamase variant with a Lys234Arg substitution, Greece, 2019. Euro Surveill. 2020;25(2):1900766. https://doi.org/10.2807/1560-7917. ES.2020.25.2.1900766

6. European Centre for Disease Prevention and Control (ECDC). Emergence of resistance to ceftazidime-avibactam in carbapenem-resistant Enterobacteriaceae - 12 June 2018. Stockholm; ECDC; 2018. Available from: https://ecdc. europa.eu/en/publications-data/rapid-risk-assessmentemergenceresistance-ceftazidime-avibactam-carbapenem

7. The European Committee on Antimicrobial Susceptibility Testing (EUCAST). Breakpoint tables for interpretation of MICs and zone diameters. Version 9.0. Växjö: EUCAST; 2019. Available from; http://www.eucast.org.

8. Galani I, Karaiskos I, Karantani I, Papoutsaki V, Maraki S, Papaioannou V, et al. On Behalf Of The Study Collaborators. Epidemiology and resistance phenotypes of carbapenemaseproducing Klebsiella pneumoniae in Greece, 2014 to 2016. Euro Surveill. 2018;23(31):1700775. https://doi.org/10.2807/15607917.ES.2018.23.30.1700775 PMID: 30086819

9. Lahiri SD, Alm RA. Identification of Novel VEB $\beta$-Lactamase Enzymes and Their Impact on Avibactam Inhibition. Antimicrob Agents Chemother. 2016;6o(5):3183-6. https://doi. org/10.1128/AAC.00047-16 PMID: 26926646

10. Galani I, Antoniadou A, Karaiskos I, Kontopoulou K, Giamarellou H, Souli M. Genomic characterization of a KPC 23-producing Klebsiella pneumoniae ST258 clinical isolate resistant to ceftazidime-avibactam. Clin Microbiol Infect. 2019;25(6):763.e5-8. https://doi.org/10.1016/j.cmi.2019.03.011 PMID: 30928562

11. Papagiannitsis CC, Giakkoupi P, Kotsakis SD, Tzelepi E, Tzouvelekis LS, Vatopoulos AC, et al. OmpK35 and 0 mpK36 porin variants associated with specific sequence types of Klebsiella pneumoniae. J Chemother. 2013;25(4):250-4. https://doi.org/10.1179/1973947813Y.0000000075 PMID: 23906079

12. Pagès JM, Peslier S, Keating TA, Lavigne JP, Nichols WW. Role of the Outer Membrane and Porins in Susceptibility of $\beta$-Lactamase-Producing Enterobacteriaceae to CeftazidimeAvibactam. Antimicrob Agents Chemother. 2016;60(3):1349-59. https://doi.org/10.1128/AAC.01585-15 PMID: 26666933

13. Mikhail S, Singh NB, Kebriaei R, Rice SA, Stamper KC, Castanheira M, et al. Evaluation of the Synergy of CeftazidimeAvibactam in Combination with Meropenem, Amikacin, Aztreonam, Colistin, or Fosfomycin against Well-Characterized Multidrug-Resistant Klebsiella pneumoniae and Pseudomonas aeruginosa. Antimicrob Agents Chemother. 2019;63(8):e0077919. https://doi.org/10.1128/AAC.00779-19 PMID: 31182535

14. Gaibani P, Lewis RE, Volpe SL, Giannella M, Campoli C, Landini MP, et al. In vitro interaction of ceftazidime-avibactam in combination with different antimicrobials against KPCproducing Klebsiella pneumoniae clinical isolates. Int J Infect Dis. 2017;65:1-3. https://doi.org/10.1016/j.ijid.2017.09.017 PMID: 28951106

15. Marshall S, Hujer AM, Rojas LJ, Papp-Wallace KM, Humphries RM, Spellberg B, et al. Can Ceftazidime-Avibactam and Aztreonam Overcome $\beta$-Lactam Resistance Conferred by Metallo- $\beta$-Lactamases in Enterobacteriaceae? Antimicrob Agents Chemother. 2017;61(4):e02243-16. https://doi. org/10.1128/AAC.02243-16 PMID: 28167541

16. Papp-Wallace KM, Winkler ML, Taracila MA, Bonomo RA. Variants of $\beta$-lactamase KPC-2 that are resistant to inhibition by avibactam. Antimicrob Agents Chemother. 2015;59(7):37107. https://doi.org/10.1128/AAC.04406-14 PMID: 25666153

17. Dubois V, Poirel L, Demarthe F, Arpin C, Coulange L, Minarini $\mathrm{LA}$, et al. Molecular and biochemical characterization of 
SHV-56, a novel inhibitor-resistant beta-lactamase from Klebsiella pneumoniae. Antimicrob Agents Chemother.

2008;52(10):3792-4. https://doi.org/10.1128/AAC.00387-08 PMID: 18663019

18. Mendonça N, Manageiro V, Robin F, Salgado MJ, Ferreira E,

Caniça M, et al. The Lys234Arg substitution in the enzyme SHV72 is a determinant for resistance to clavulanic acid inhibition. Antimicrob Agents Chemother. 2008;52(5):1806-11. https:// doi.org/10.1128/AAC.01381-07 PMID: 18316518

19. Mendonça N, Ferreira E, Louro D, Caniça M, Caniça MARSIP Participants. Molecular epidemiology and antimicrobial susceptibility of extended-and broad-spectrum betalactamase-producing Klebsiella pneumoniae isolated in Portugal. Int J Antimicrob Agents. 2009;34(1):29-37. https:// doi.org/10.1016/j.ijantimicag.2008.11.014 PMID: 19272757

20. Manageiro V, Ferreira E, Albuquerque L, Bonnet R, Caniça M. Biochemical study of a new inhibitor-resistant beta-lactamase, SHV-84, produced by a clinical Escherichia coli strain.

Antimicrob Agents Chemother. 2010;54(5):2271-2. https://doi. org/10.1128/AAC.01442-09 PMID: 20211886

21. Galani I, Nafplioti K, Adamou P, Karaiskos I, Giamarellou $\mathrm{H}$, Souli MStudy Collaborators. Nationwide epidemiology of carbapenem resistant Klebsiella pneumoniae isolates from Greek hospitals, with regards to plazomicin and aminoglycoside resistance. BMC Infect Dis. 2019;19(1):167. https://doi.org/10.1186/s12879-019-3801-1 PMID: 30770727

\section{License, supplementary material and copyright}

This is an open-access article distributed under the terms of the Creative Commons Attribution (CC BY 4.0) Licence. You may share and adapt the material, but must give appropriate credit to the source, provide a link to the licence and indicate if changes were made.

Any supplementary material referenced in the article can be found in the online version.

This article is copyright of the authors or their affiliated institutions, 2020. 